\title{
Loss of heterozygosity for DNA polymorphisms mapping to chromosomes 10 and 17 and prognosis in patients with gliomas
}

\author{
C E Jones, M B Davis, J L Darling, J F Geddes, D G T Thomas, A E Harding
}

\begin{abstract}
Twenty nine patients with gliomas were investigated for loss of heterozygosity for 40 DNA polymorphisms in tumour DNA, particularly concentrating on those mapping to chromosomes 10 and 17. Eight of 18 grade IV gliomas showed loss of sequences from chromosomes 10,17 , or both. The data suggested total loss of one copy of chromosome 10 , but there were interstitial deletions of the short arm of chromosome 17 in three of five tumours. Heterogeneous interstitial deletions of chromosome 17 were also found in two lower grade astrocytomas and one benign oligodendroglioma. The striking finding of this study was that patients with high grade gliomas whose tumours exhibited loss of heterozygosity for chromosomes 10,17 , or both survived significantly longer after surgery (median $17 \cdot 4$ months) than those whose tumours did not show loss of these chromosomes (median 6.7 months). These findings suggest that there is a subset of particularly aggressive high grade gliomas with no currently known molecular genetic abnormalities.
\end{abstract}

(F Neurol Neurosurg Psychiatry 1995;58:218-221)

Keywords: gliomas; DNA polymorphisms; prognosis

Gliomas are the most common primary tumours of the nervous system and there is evidence that the genetic mechanisms involved in their pathogenesis are complex. They exhibit various cytogenetic abnormalities, including polyploidy and aneuploidy, but also a tendency to show specific chromosomal loss, particularly for chromosome $10 .^{1}$ It is possible to detect loss of DNA sequences in tumours by assessing the maintenance or loss of heterozygosity ( $\mathrm{LOH}$ ) in tumour DNA compared with that from the patient's blood. This can be most easily demonstrated if genomic DNA from the patient is heterozygous for a given restriction fragment length polymorphism (RFLP), but one allele is missing from tumour DNA. With this approach, which is more sensitive for small chromosomal deletions, it has been shown that $\mathrm{LOH}$ for sequences mapping to chromosome 10 is common in glioblastomas. ${ }^{28}$ Losses of chromosome 17, particularly the short arm, occur in all grades of astrocytomas. ${ }^{9-14}$ Other chro- mosomal regions lost in some adult astrocytomas include $9 \mathrm{p}, 13 \mathrm{q}, 19 \mathrm{q}$, and $22 \mathrm{q} .^{2415-18} \mathrm{It}$ has been suggested that survival time is better in patients whose gliomas have normal karyotypes on cytogenetic analysis than those with tumours showing clonal abnormalities, ${ }^{19}$ but the relation, if any, between prognosis and molecular genetic abnormalities has not been reported. In this study we have investigated a total of 29 gliomas for LOH of DNA sequences, particularly concentrating on chromosomes 10 and 17 . In addition, we have correlated the presence or absence of $\mathrm{LOH}$ with clinical outcome.

\section{Patients and methods}

Samples of tumours were obtained from 29 patients with gliomas of a variety of histological grades at the time of debulking surgical procedures performed between February and October 1988. These samples were adjacent to those analysed histologically. DNA was extracted from carefully dissected and washed tumour tissue and simultaneously obtained blood samples by standard methods. Approximately $3 \mu \mathrm{g}$ of DNA from each sample was digested separately with MspI, TaqI, PvuII, PstI, EcoRI, and HindIII. The digested DNA was electrophoresed through horizontal agarose gels, with tumour and blood DNA samples from each patient in adjacent lanes, and transferred to nylon membranes (Hybond-N, Amersham) by Southern blotting before hybridisation to probes labelled with ${ }^{32} \mathrm{P}$. These included eight mapping to chromosome 10, 13 (detecting 14 RFLPs) mapping to $17 \mathrm{p}$, and one to $17 \mathrm{q}$ (figure). RFLPs were also studied with a further 18 probes mapping to 15 other chromosomes: D1S7 (1p35-33), MST1 (3p21), D4S10 and D4S95 (4p16.3), D5S43 (5q35-qter), pHLA1·1 (6p21.3), D7S23 (7q31-32), D9S10 (9q34-33), NRASL1 (9p12-21), D11S97 (11q13.1), GLI (12q13), RB1 (13q14.3), D15S86 (15q26), D16S85 (16p13.3), LDLR (19p13.2), D19S9 (19q13.1), D20S26 (20q), and D22S164 $(22 q) .^{20}$ After hybridisation filters were exposed to Fuji-XR film for 24 hours to seven days at $-70^{\circ} \mathrm{C}$; hybridisation patterns of tumour and blood DNA were compared on the resulting autoradiographs.

The tumour sections were graded histologically by one neuropathologist (JFG), as oligodendroglioma and astrocytoma grades I-IV as defined by Daumas-Duport and colleagues. ${ }^{21}$ 
Chromosome 10 loci and patterns of loss of heterozygosity

Tumour number

\begin{tabular}{|l|l|c|c|c|c|c|c|c|c|}
\hline Band & Locus & 49 & 35 & 20 & 36 & 33 & 23 & 32 & 2 \\
\hline \multirow{2}{*}{ 15 } & D10S92 & $\bullet$ & $\Delta$ & $\bullet$ & $\bullet$ & $\Delta$ & $\bullet$ & $\Delta$ & $\Delta$ \\
\cline { 2 - 10 } & D10S17 & ND & ND & $\Delta$ & ND & ND & $\bullet$ & ND & ND \\
\hline q11.2 & D10S15 & $\Delta$ & $\Delta$ & ND & ND & $\Delta$ & $\Delta$ & $\Delta$ & $\Delta$ \\
\hline q21.1 & D10S22 & $\Delta$ & ND & $\Delta$ & ND & ND & $\bullet$ & ND & $\bullet$ \\
\hline q21.1-22 & D10S19 & $\bullet$ & $\Delta$ & $\Delta$ & $\Delta$ & $\Delta$ & ND & $\Delta$ & $\Delta$ \\
\hline q23.3 & GLUD & ND & $\Delta$ & $\Delta$ & $\Delta$ & $\bullet$ & $\Delta$ & $\Delta$ & $\Delta$ \\
\hline q26 & D10S25 & ND & ND & ND & $\Delta$ & ND & $\bullet$ & ND & $\Delta$ \\
& D10S90 & $\bullet$ & $\bullet$ & $\Delta$ & $\Delta$ & $\bullet$ & ND & $\bullet$ & $\bullet$ \\
\hline
\end{tabular}

Chromosome 17 loci and patterns of loss of heterozygosity

Tumour number/type

\begin{tabular}{|c|c|c|c|c|c|c|c|c|c|}
\hline Band & Locus & 49 & 35 & 33 & 50 & 12 & Olig & Astro I & Astro III \\
\hline \multirow[t]{2}{*}{ p13.3 } & D17S5 & $\bullet \wedge$ & 0 & $\bullet \wedge$ & $\Delta \Lambda$ & ND $\wedge$ & $\Delta$ & $\Delta$ & $\bullet \wedge$ \\
\hline & D17S28 & $\bullet$ & 0 & $\bullet$ & $\Delta$ & $\Delta$ & $\Delta$ & 0 & $\Delta$ \\
\hline \multirow[t]{3}{*}{ pter-12 } & D17S126 & $\Delta$ & ND & ND & $\Delta$ & $\Delta$ & 0 & $\Delta \Lambda$ & $\Delta$ \\
\hline & D17S68 & ND & 0 & ND & ND & ND & $\Delta$ & $\bullet$ & $\Delta$ \\
\hline & D17S66 & $\Delta$ & $\Delta \wedge$ & ND & $\Delta$ & ND & $\Delta$ & $\Delta V$ & $\Delta$ \\
\hline p13.1 & TP53 & $\Delta$ & $\Delta$ & ND & $\Delta$ & ND & $\Delta$ & 0 & $\Delta$ \\
\hline pter-p12 & D17S67 & ND & ND & ND & ND & ND & ND & 0 & ND \\
\hline \multirow[t]{5}{*}{ p12-11.2 } & D17S124 & ND V & $\bullet$ & ND & ND & ND & 0 & $\Delta$ & $\Delta V$ \\
\hline & D17S125 & 0 & $\Delta$ & ND & - V & ND & $\bullet \wedge$ & 0 & 0 \\
\hline & D17S122 & 0 & ND & ND & 0 & ND & $\Delta$ & ND & $\Delta$ \\
\hline & D17S71 & 0 & $\Delta$ & ND & $\Delta$ & $\Delta$ & $\Delta$ & $\Delta$ & $\Delta$ \\
\hline & D17S61* & $\Delta$ & $\Delta$ & ND & 0 & ND & $\Delta V$ & ND & $\Delta$ \\
\hline p11.2-cen & D17S58 & $\Delta$ & $\Delta$ & ND & $\Delta$ & $\bullet$ & 0 & 0 & $\Delta$ \\
\hline q11.2-12 & THRA1 & $\Delta$ & $\Delta V$ & ND V & $\Delta$ & $\Delta V$ & $\Delta$ & 0 & $\Delta$ \\
\hline
\end{tabular}

Figure Chromosomes 10 and 17 polymorphisms and patterns of loss of heterozygosity in different tumours.

Locus order ${ }^{20}$ is from distal short arm (top) to distal long arm (bottom). Loci in the same box have not been mapped relative to each other; ${ }^{\star}$ not mapped relative to other loci in the p12-11.2 group.Tumour numbers are as in the table. ND $=$ not done; $\mathrm{O}=\mathrm{LOH}, \boldsymbol{\Delta}=$ not informative; $\mathrm{O}=$ herozygosity maintained; $\wedge$ and $\vee=$ possible upper and lower limits of deletions respectively; these extend to, but do not include, immediately adjacent loci. Olig = benign oligodendroglioma; Astro I and III = astrocytomas grades I and III.

Statistical comparisons were performed with the Mann-Whitney $U$ test, Spearman's rank correlation, and $\chi^{2}$ analysis with Yates' correction for $2 \times 2$ tables.

\section{Results}

Eighteen patients had grade IV astrocytomas.

Table Clinical and molecular genetic features of patients with grade IV astrocytomas

\begin{tabular}{|c|c|c|c|c|c|c|c|c|}
\hline $\begin{array}{l}\text { Patient } \\
\text { no }\end{array}$ & $\begin{array}{l}\text { Age } \\
(y)\end{array}$ & $\begin{array}{l}\text { Site of } \\
\text { tumour }\end{array}$ & $\begin{array}{l}\text { Karnofsky } \\
\text { score }\end{array}$ & $\begin{array}{l}\text { Onset to } \\
\text { surgery } \\
\text { (months) }\end{array}$ & $\begin{array}{l}\text { Surgery to } \\
\text { death } \\
\text { (months) }\end{array}$ & Radiotherapy & Chemotherapy & $y \mathrm{LOH}$ \\
\hline 2 & 61 & RP-O & 90 & 2 & 13 & + & - & 10 \\
\hline 4 & 59 & RO & $60-70$ & 2 & 13 & + & + & - \\
\hline 11 & 68 & RF-P & 60 & 6 & 5 & + & - & - \\
\hline 12 & 54 & RF & 80 & 1 & 13 & + & + & $17^{\star}$ \\
\hline 20 & 38 & R F-P & 80 & 3 & 23 & + & + & 10 \\
\hline 21 & 30 & RT & 40 & $6(y)$ & 12 & - & - & - \\
\hline 22 & 54 & $\mathbf{R P}$ & 50 & 1 & 7 & + & + & - \\
\hline 23 & 65 & RT & 50 & 1 & 8 & + & - & 10 \\
\hline 24 & 55 & RF & 30 & 2 & 3 & - & - & - \\
\hline 32 & 56 & RF & 40 & 1 & 9 & + & + & 10 \\
\hline 33 & 57 & LT-P & 80 & 1 & 14 & + & - & 10,17 \\
\hline 35 & 44 & $\mathrm{LP}$ & 40 & 1 & 18 & + & + & 10,17 \\
\hline 36 & 39 & $\overrightarrow{R P}$ & 80 & 3 & 40 & + & + & 10 \\
\hline 44 & 64 & LP & $40-50$ & 1 & 1 & - & - & - \\
\hline 47 & 50 & LF & 40 & 3 & 3 & + & + & - \\
\hline 48 & 58 & LT & $20-30$ & 1 & 10 & + & - & - \\
\hline 49 & 65 & RT & 30 & 1 & 6 & + & + & 10,17 \\
\hline 50 & 26 & LF & 90 & 4 & $60 t$ & + & + & $17^{\star}$ \\
\hline
\end{tabular}

${ }^{\star}$ Not informative for chromosome 10 markers; talive; $L=$ left; $R=$ right; $F=$ frontal; $P=$ parietal; $\mathrm{T}=$ temporal; $\mathrm{O}=$ occipital.
All but two of these were informative for one or more loci on chromosome 10 and all were informative for one or more chromosome 17 loci. The table shows the numbers of tumours with 10 or 17 losses. The pattern of $\mathrm{LOH}$ of chromosome 10 sequences indicated that the whole of this chromosome was lost in these tumours (figure); there was no evidence for maintenance of heterozygosity for some chromosome $\mathbf{1 0}$ markers and loss for others in any of them. By contrast, data for chromosome 17 indicate partial loss of the short arm in some tumours (figure). Eleven lower grade tumours were investigated: one grade I, six grade II, and, one grade III astrocytomas, and three oligodendrogliomas. The grade I astrocytoma showed partial loss of heterozygosity for chromosome 17, as did the grade III astrocytoma and one benign oligodendroglioma (figure).

LOH for RFLPs mapping to other chromosomes was detected in four tumours. One of these, a grade IV astrocytoma (case 49), had lost heterozygosity for sequences from chromosomes 19p and 22q. The other losses were in the grade III astrocytoma (1p), and two grade IV astrocytomas (case $33 ; 4 p$ and case 12; 9q). One grade IV tumour (case 11) showed amplification of the gli oncogene, 
demonstrated by a marked increase in hybridisation signal.

There were too few patients with low grade tumours in each category of malignancy, or with chromosomal losses, to permit correlation between molecular genetic data and prognosis. In patients with grade IV astrocytomas, table 2 shows the relevant clinical features. Most were treated by radiotherapy, and in many cases adjuvant chemotherapy, after surgery. Only one patient is still alive, 60 months after her operation.

In patients whose tumours showed $\mathrm{LOH}$ for chromosomes 10,17 , or both, median survival after surgery was $17 \cdot 4$ (range 6-60) months, compared with $6 \cdot 75$ (1-13) months in patients whose tumours did not show loss of sequences from these chromosomes ( $p=$ $0 \cdot 01)$. There was still a significant difference in survival if the two patients with tumours uninformative for chromosome 10 markers were excluded $(p<0.05)$. Seventeen of the 18 patients with high grade gliomas had surgery within six (usually within three) months of onset of symptoms (table 2). The exception (patient 21) presented with seizures six years previously and had a temporal lobectomy followed by radiotherapy two years later. Median duration of symptoms before surgery was one (range 1-4) month in the LOH group and two (range 1-72) months in the patients with no LOH $(p>0.05)$. There was no difference in the age distribution of patients whose tumours showed $\mathrm{LOH}$ (median 55, range 26-65 years), compared with those whose did not (median 56.5, range 30-68 years, p > $0.05)$. The LOH group had higher Karnofsky scores at presentation (median 80, range 30-90, compared with $42 \cdot 5$, range 25-65), although this difference was not statistically significant $(p>0 \cdot 05)$. Therapeutic decisions reflected this; all the patients with $\mathrm{LOH}$ had postoperative radiotherapy, with or without chemotherapy, whereas three of the other eight had neither (table $2 ; \chi^{2}=2 \cdot 2, \mathrm{p}=0 \cdot 14$ ). There was no correlation between age and Karnofsky score in all the patients with grade IV tumours $(r=-0 \cdot 18, \mathrm{p}=0 \cdot 46)$. There was a significant inverse correlation between age and survival $(r=-0.56, \mathrm{p}=0.016)$, and a positive correlation between Karnofsky score and postoperative survival $(r=0.59, \mathrm{p}=$ $0 \cdot 01$ ).

\section{Discussion}

Our molecular genetic data are in agreement with those of others in that $\mathrm{LOH}$ for chromosome 10 loci was restricted to grade IV gliomas, in eight of 16 informative samples $(50 \%)$. The frequency of this finding has varied substantially, from $51 \%$ to $95 \%$ in previous studies. ${ }^{23}$ 5-810 This presumably reflects differences in number, informativeness, and localisation of probes used, and some variation in pathological classification. As was the case in this series, evidence for partial, as opposed to total, loss of chromosome 10 is relatively rare. Some authors have described occasional LOH for only $10 \mathrm{p}$ or $10 \mathrm{q}$, or reten- tion of only the distal part of 10q. ${ }^{48}$ Fujimoto and colleagues ${ }^{3}$ defined a common region of loss on chromosome 10 in three tumours with interstitial deletions, between the middle of the short arm and band 10q23. These data are compatible with observations in two tumours retaining part of distal $10 q,{ }^{4}$ suggesting the presence of a tumour suppressor gene in this region that relates to high grade malignancy in gliomas.

By contrast with chromosome 10 , partial loss of chromosome 17, particularly involving the distal short arm (17p11.1-pter) is common and occurs in all grades of astrocytoma, as found here. We found $\mathrm{LOH}$ for chromosome 17 in eight of $24(33 \%)$ informative astrocytomas within the range of frequencies reported previously, $11-50 \% .^{8-14}$ All three informative grade IV gliomas with $\mathrm{LOH}$ for chromosome 17 had also lost sequences from chromosome 10; this combination is known to occur in $25 \%$ to $50 \%$ of grade IV tumours with $\mathrm{LOH}$ for chromosome 10, and suggests that these tumours may represent a subset that arises through progression from lower grade astrocytomas. ${ }^{89}$

James and colleagues ${ }^{11}$ suggested that $\mathrm{LOH}$ for chromosome 17 was confined to tumours of astrocytic differentiation, but one benign oligodendroglioma in this series had an interstitial deletion of one copy of $17 p$ (figure). Relatively few molecular genetic data concerning oligodendrogliomas have been reported; James and coworkers studied six oligodendrogliomas but used fewer chromosome 17 probes than in the present study. $\mathrm{LOH}$ for $19 \mathrm{q}$ sequences has been described in four of seven oligodendrogliomas. ${ }^{7}$

The deletions of chromosome $17 \mathrm{p}$ seen in astrocytomas often involve the p53 tumour suppressor gene. This gene has been implicated in the pathogenesis of a wide variety of cancers, and mutations of p53 are found in about $30 \%$ of diffuse fibrillary astrocytomas, usually accompanied by loss of the other copy of chromosome $17 \mathrm{p}$, which is to be expected given the recessive nature of tumour suppressor genes ${ }^{14}$; however, p53 point mutations have been described without loss of the other $17 p,{ }^{121421}$ and some losses of $17 p$ do not involve the p53 locus. ${ }^{13}$ The chromosome 17 deletions in six of our tumours may include p53, but one (a grade I astrocytoma) maintained heterozygosity for p53 with $\mathrm{LOH}$ for a more distal $17 \mathrm{p}$ locus (figure). These findings suggest that there is another, more telomeric, tumour suppressor gene on chromosome $17 \mathrm{p}$, in this case between p53 and D17S28. The data from the oligodendroglioma indicate yet a further deleted region of $17 \mathrm{p}$ between DS58 and DS124 (figure). We did not investigate other chromosomes systematically, but found $\mathrm{LOH}$ in three tumours for chromosomes $1 \mathrm{p}$, $4 \mathrm{p}$, or $9 \mathrm{q}$. Occasional losses of $1 \mathrm{p}$ and $9 \mathrm{q}$, and other chromosomal regions, have been noted before, ${ }^{4918}$ and are probably not of any pathogenetic relevance. We did not find any tumours with $\mathrm{LOH}$ for RB1, which has previously been reported in four of nine glioblastomas, ${ }^{17}$ or $19 q$ or $22 q$, seen in apparently 
non-random proportions of other series of gliomas. $^{22} 23$ One grade IV tumour showed evidence of amplification of $g l i$, an oncogene that maps to chromosome 12q; this has been reported in only occasional gliomas to date. ${ }^{23} 24$

The striking finding in this study is the association between LOH and longer postoperative survival, more than twice as long as those without $\mathrm{LOH}$, in patients with high grade gliomas, despite the small numbers. This may well be explained by the tendency for patients whose tumours showed $\mathrm{LOH}$ to have higher Karnofsky scores at presentation, although this was not statistically significant. There was no difference in duration of preoperative symptoms. There was a correlation between Karnofsky status and survival in this series of grade IV tumours overall, as has been noted previously. ${ }^{25}$ Although age is known to be a major determinant of survival in patients with malignant gliomas ${ }^{25} 26$ and was inversely correlated with survival in this series, there was no difference in age between the two groups of patients defined by presence or absence of $\mathrm{LOH}$. One possible explanation for longer survival in the $\mathrm{LOH}$ group is that slightly, but not significantly, more had postoperative radiotherapy, and often adjuvant chemotherapy, than in those without LOH. This was largely determined by Karnofsky status.

The explanation for this finding is not clear. It is unexpected, given recent observations made by von Deimling and colleagues. ${ }^{8}$ Based on a series of 67 patients with glioblastomas, these authors suggested that two main genetic types could be defined. One showed $\mathrm{LOH}$ for chromosome 17 and sometimes for chromosome 10, but did not exhibit amplification of the epidermal growth factor receptor (EGFR) gene. These tumours, which may be derived from lower grade astrocytomas, tended to occur in younger female patients. Amplification of EGFR was only found in tumours with LOH for chromosome 10 , but not all of them, and rarely in those with additional LOH for chromosome 17. Patients with this second type of tumour exhibiting EGFR amplification were, on average, older and more likely to be male. Given that age is a major determinant of prognosis in glioblastoma, it might be expected that patients with tumours showing LOH for chromosome 10 would have a particularly poor prognosis. Von Deimling and coworkers ${ }^{8}$ did not analyse demographic data from their patients without LOH for chromosomes 10 or 17 , who had a particularly bad prognosis in our series, although the age range of these patients, like ours, was wide. Our findings, which need to be confirmed, suggest that there is a subset of high grade gliomas, with currently no known molecular genetic substrate, which is particularly aggressive.
We thank the Brain Research Trust for financial support and Drs B Vogelstein, J Armour, and B Carritt for providing probes.

1 Bigner SH, Mark J, Burger PC, et al. Specific chromosomal abnormalities in malignant human gliomas. Cancer Res 1988;88:405-11.

2 James CD, Carlbom E, Dumanski JP, et al. Clonal genomic alterations in glioma malignancy stages. Cancer Res 1989;48:5546-51.

3 Fujimoto M, Fults DW, Thomas GA, et al. Loss of heterozygosity on chromosome 10 in human glioblastoma multiforme. Genomics 1989;4:210-14.

4 Fults D, Pedone CA, Thomas GA, White R. Allelotype of human malignant astrocytoma. Cancer Res 1990;50: 5784-9.

5 Watanabe $K$, Nagai $M$, Wakai S, Arai T, Kawashima $K$. Loss of constitutional heterozygosity in chromosome 10 in human glioblastoma. Acta Neuropathol 1990;80: in hum.

6 Venter DJ, Thomas DGT. Multiple sequential molecular abnormalities in the evolution of human gliomas. $\mathrm{Br} f$ Cancer 1991;63:753-7.

7 von Deimling A, Louis DN, von Ammon $\mathrm{K}$, et al. Association of epidermal growth factor receptor gene amplification with loss of chromosome 10 in human glioblastoma multiforme. F Neurosurg 1992;77:295-301.

8 von Deimling A, von Ammon K, Schoenfeld D, Wiestler OD, Seizinger BR, Louis DN. Subsets of glioblastoma multiforme defined by molecular genetic analysis. Brain Pathol 1993;3:19-26.

9 James CD, Carlbom E, Nordenskjold M, Collins VP, Cavenee WK. Mitotic recombination of chromosome 17 in astrocytomas. Proc Natl Acad Sci USA 1989;86: in astrocy.t.

10 Fults D, Tippets RH, Thomas GA, Nakamura Y, White $R$. Loss of heterozygosity for loci on chromosome $17 p$ in human malignant astrocytoma. Cancer Res 1989;49: 6572-7.

11 El-Azouzi M, Chung RY, Farmer GE, et al. Loss of distinct regions on the short arm of chromosome 17 associated with tumorigenesis of human astrocytomas. Proc Natl Acad Sci USA 1989;86:7186-90.

12 Frankel RH, Bayona W, Koslow M, Newcomb EW. p53 mutations in human malignant gliomas: comparison of loss of heterozygosity with mutation frequency. Cancer Res 1992;52:1427-33.

13 Saxena A, Clark WC, Robertson JT, Ikejiri B, Oldfield $\mathrm{EH}, \mathrm{Ali}$ IU. Evidence for the involvement of a potential second tumor suppressor gene on chromosome 17 distinct from p53 in malignant astrocytomas. Cancer Res tinct from p53 in

14 von Deimling A, Eibl RH, Ohgaki $\mathrm{H}$, et al. p53 mutations are associated with $17 \mathrm{p}$ allelic loss in grade II and grade III astrocytoma. Cancer Res 1992;52:2987-92.

15 von Deimling A, Louis DN, von Ammon K, Petersen I, Wiestler OD, Seizinger BR. Evidence for a tumour suppressor gene on chromosome $19 \mathrm{q}$ associated with human astrocytomas, oligodendrogliomas, and mixed gliomas. Cancer Res 1992;52:4277-9.

16 Rey JA, Bello MJ, Jimenez-Lara AM, et al. Loss of heterozygosity for distal markers on $22 \mathrm{q}$ in human gliomas. Int $\mathcal{f}$ Cancet 1992;51:703-6.

17 Venter DJ, Bevan KL, Ludwig RL. Retinoblastoma gene deletions in human glioblastomas. Oncogene 1991;6: 445-8

18 Bello MJ, de Campos JM, Vaquero J, et al. Molecular and cytogenetic analysis of chromosome 9 deletions in 75 malignant gliomas. Genes, Chromosomes, and Cancer 1994;9:33-41.

19 Kimmel DW, O'Fallon JR, Scheithauer BW, Kelly PJ, Dewald GW, Jenkins RB. Prognostic value of cytogenetic analysis in human cerebral astrocytomas. Ann Neurol 1992;31:534-42.

20 NIH/CEPH Collaborative Mapping Group. A comprehensive genetic linkage map of the human genome. Science 1992;258:67-86.

21 Daumas-Duport C, Scheithauer B, O'Fallon J, Kelly P. Grading of astrocytomas: a simple and reproducible method. Cancer 1989;62:2152-65.

$22 \mathrm{Wu} \mathrm{JK}$, Ye Z, Darras BT. Frequency of p53 tumour suppressor gene mutations in human primary brain tumors.

23 Kinzler KW, Bigner SH, Bigner DD, et al. Identification of an amplified, highly expressed gene in a human glioma. Science 1987;236:70-3.

24 Fuller GN, Bigner SH. Amplified cellular oncogenes in neoplasms of the human central nervous system. Mutat Res 1992;276:299-306.

25 Walker MD, Alexander E, Hunt WE, et al. Evaluation of $\mathrm{BCNU}$ and/or radiotherapy in the treatment of anaplastic gliomas. f Neurosurg 1978;49:333-43.

26 Medical Research Council Brain Tumour Working Party. Prognostic factors for high-grade malignant glioma: development of a prognostic index. F Neurooncol 1990; 9:47-55 